\title{
Chronic cough in Swiss bagpipe player
}

Schindler, Valeria ; Gubler, Christoph ; Turk, Alexander ; Becker, Anton S ; Pohl, Daniel

DOI: https://doi.org/10.1136/gutjnl-2017-315420

Posted at the Zurich Open Repository and Archive, University of Zurich

ZORA URL: https://doi.org/10.5167/uzh-144798

Journal Article

Accepted Version

Originally published at:

Schindler, Valeria; Gubler, Christoph; Turk, Alexander; Becker, Anton S; Pohl, Daniel (2018). Chronic cough in Swiss bagpipe player. Gut, 67(10):1792.

DOI: https://doi.org/10.1136/gutjnl-2017-315420 


\section{Chronic Cough in Swiss Bagpipe Player}

Valeria Schindler ${ }^{1}$, Christoph Gubler ${ }^{1}$, Alexander Turk², Anton S. Becker ${ }^{3}$, Daniel Pohl ${ }^{1}$

${ }^{1}$ Division of Gastroenterology and Hepatology, University Hospital Zurich, Zurich, Switzerland; ${ }^{2}$ Department of Internal Medicine, See-Spital Horgen, Horgen, Switzerland; ${ }^{3}$ Institute of Diagnostic and Interventional Radiology, University Hospital Zurich, Zurich, Switzerland

Corresponding Author's Contact Information:

Dr. med. Valeria Schindler

Division of Gastroenterology and Hepatology

University Hospital Zurich

Raemistrasse 100

8091 Zurich

Switzerland

E-Mail: valeria.schindler@usz.ch

Tel: +41442551111

Key words: gastroesophageal reflux disease, esophageal pH monitoring, esophageal reflux, esophageal motility, anti-reflux therapy

Word count "introduction": 130

Word count "answer": 207

Abbreviations:

24h-MII-pH

24h-pH-multichannel intraluminal impedance testing

BAL bronchoalveolar lavage

CT computed tomography

GERD gastroesophageal reflux disease

GI gastrointestinal

HRM high resolution esophageal manometry 


\section{Introduction:}

A 34-year-old man presented to our gastrointestinal (GI) clinic with chronic cough. Coughing episodes exacerbated on days of bagpipe playing and the morning after. Methacholine testing revealed airway hyperreactivity. Allergic bronchopulmonary aspergillosis could be excluded; computed tomography (CT) scan of the chest was without pathological findings. Also bronchoscopy with bronchoalveolar lavage (BAL) and mucosal biopsy failed to reveal a pulmonary etiology of the cough. Minor findings in BAL were an elevated number of macrophages and bacteria representative of the oral flora. A trial with inhalative Budesonide and Formoterol and consecutively, 8 weeks of PPI treatment were started, however only lead to inadequate reduction of symptoms. The patient was then referred to us, where we performed high resolution esophageal manometry (HRM) and 24h-pH-multichannel intraluminal impedance testing (24h-MII-pH) after unremarkable upper endoscopy.

\section{Answer:}

During bagpipe playing abdominal/esophageal pressures increased simultaneously to 80 90mmHg Figure 1 above and Video. 24h-MII-pH demonstrated gastroesophageal reflux disease (GERD, time of acid exposure, distal esophagus: 7.2\% [norm <4.2\%], DeMeester 24.1 $[<14.7])$. Testing was also performed during 10min bagpipe playing Figure 1 below: Two reflux episodes were noticed, followed by elevated acid exposure time of the distal esophagus. Instead of choosing acid-suppressive therapy, the patient decided to modify his bagpipe (replacement of internal bag with smaller bag, changing of reeds to lower resistance).

Upon follow-up appointment seven months later the patient reported resolution of cough. During repeat HRM while bagpipe playing abdominal/esophageal pressures were reduced by $30 \%$ (probably also by reduction of strain on the crural diaphragm) and criteria for GERD were no longer fulfilled during 24h-MII-pH (acid exposure: 2.0\%, DeMeester 6.0) Figure 2. In fact, no episodes of reflux occurred in the $10 \mathrm{~min}$ bagpipe playing period or in the first hour thereafter on 24h-MII-pH. 
This case demonstrates a patient with chronic cough due to GERD triggered by bagpipe playing. Subjective and objective findings were resolved only by bagpipe reconstruction without acid-suppressive medication or additional lifestyle changes. A gastrointestinal cause of symptoms should be considered in patients playing a wind instrument with inconclusive pulmonary work-up for cough.

Conflicts of interest:

Valeria Schindler: support from Swiss National Foundation (SNF, grant funding) for research but no financial relationships or activities with any organizations that might have an interest in the submitted work.

Christoph Gubler: none

Alexander Turk: none

Anton S. Becker: none

Daniel Pohl: none 
Figure 1: High-resolution manometry (HRM) and 24-hour multichannel intraluminal impedance-pH (24h-MII-pH) testing while bagpipe playing before reconstruction. 


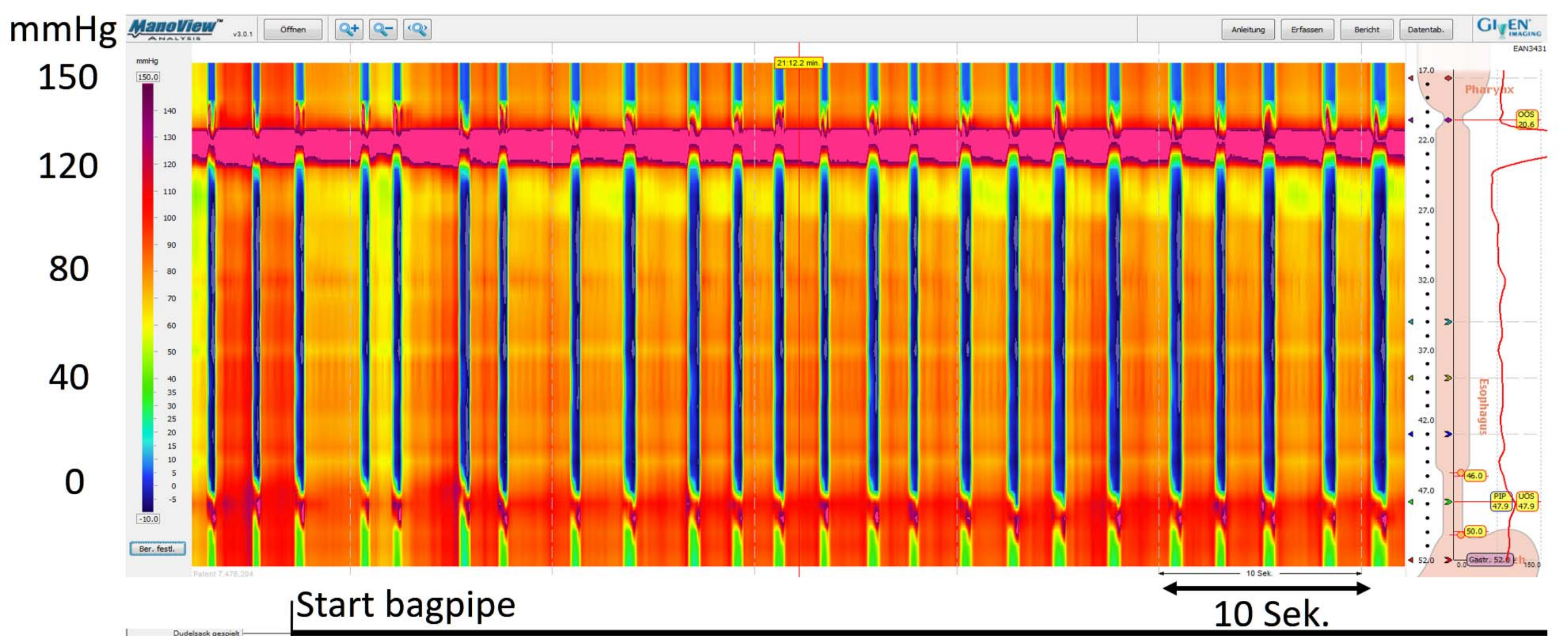

\section{1: Z Body}

\section{3: Z Body}

\section{6: Z Body}

7: pH Body

8: pH Gastric

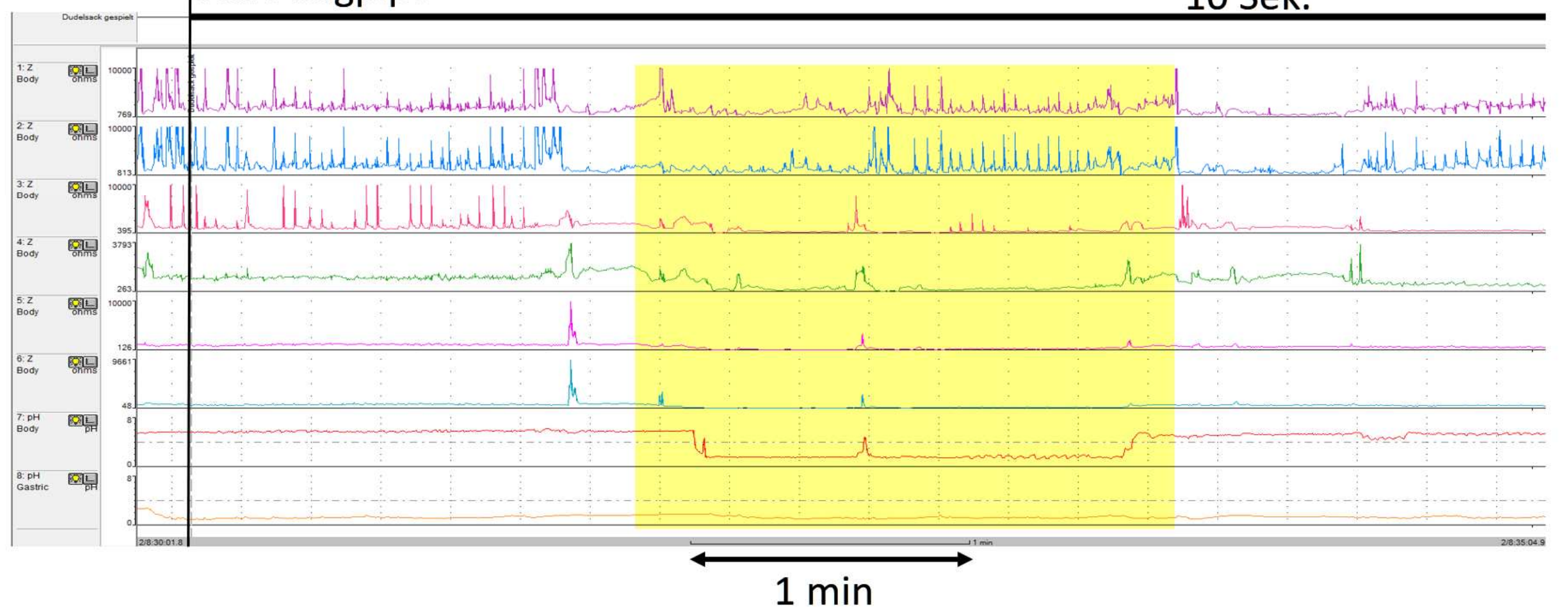


Figure 2: High-resolution manometry (HRM) and 24-hour multichannel intraluminal impedance-pH (24h-MII-pH) testing while bagpipe playing after reconstruction. 
